\title{
sciendo
}

\section{Regulation of Stride Length During the Approach Run in the 400-M Hurdles}

\author{
by

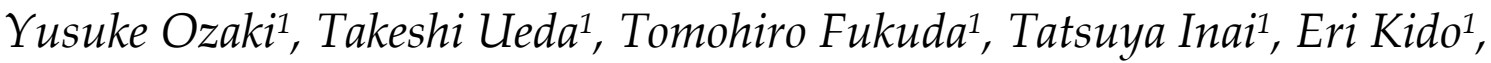 \\ Daiki Narisako ${ }^{1}$
}

This research aims to clarify the stride adjustment in the approach of the 400-m hurdles, and to examine the relationship with 400-m hurdle performance. Seven male 400-m hurdlers volunteered for this study. Participants ran three times from the start to the second hurdle. The standard deviation of toe-hurdle distance and standard deviation of stride length at each step from the start to the first hurdle were calculated. The maximum value of the standard deviation of toe-hurdle distance was defined as the position at which the athlete starts stride adjustment. The relationships between each variable, 400-m hurdle personal best, and the ratio of 400-m hurdle personal best and 400- $m$ running personal best (400 m/400-m hurdles) were examined. Results concluded that standard deviation of toe-hurdle distance gradually increased after the start, reached the maximum value in the latter half of the approach section, and then decreased until the takeoff. Standard deviation of stride length increased significantly from 4 steps before the takeoff. From these trends, it was suggested that athletes seemed to start stride adjustment from the middle stage to the latter half of the approach by sensing stride error accumulation in the middle of the approach. The strides immediately before the takeoff were markedly involved in stride adjustment. Furthermore, the stride adjustment technique to reduce maximum accumulation error of stride evaluated in the approach section was considered associated with the smooth running of the entire 400-m hurdle race.

Key words: hurdles, maximum accumulation error, stride length, standard deviation of toe-hurdle distance.

\section{Introduction}

The 400-m hurdles (400 m-H) event involves running while clearing 10 hurdles installed at 35$\mathrm{m}$ intervals. It requires different techniques compared with the 110-m hurdle event, such as hurdle clearance on a curved track, regulation of stride length, pacing, and stride patterns. Undoubtedly, performance on the $400 \mathrm{~m}-\mathrm{H}$ is related to the running ability in a 400-m run. However, Iskra and Pietrzak (2016) indicated that even world-class $400 \mathrm{~m}-\mathrm{H}$ athletes do not have the best personal records at 400-m runs. Therefore, $400 \mathrm{~m}-\mathrm{H}$ specific techniques, such as stride adjustment and hurdle clearance on curves, are considered important.

The maximum velocity during the race occurs between the first and third hurdle, and then it decreases gradually (Ditroilo, 2001; Morioka et al., 2005; Nagasawa, 1995; Yasui et al., 2008). Therefore, it can be judged that the approach section, which is the acceleration phase from the start to the first hurdle, is an important phase for increasing the maximum velocity. Indeed, there is a strong correlation between maximum velocity, the velocity in the approach, and performance in the $400 \mathrm{~m}-\mathrm{H}$ (Yasui et al., 2008). In addition, Karube et al. (2003) stated that athletes emphasized the first hurdle regardless of the performance level and sex, and the sense of the athlete also showed the importance of the approach. In this approach section, the athletes accelerate and adjust the stride to the ideal take

1 - Graduate School of Education, Hiroshima University, Higashihiroshima, Japan. 
off position to clear the first hurdle located on the curve. At that time, athletes must maintain their speed to achieve high performance throughout the race. Previous studies on the $400 \mathrm{~m}-\mathrm{H}$ are mostly related to hurdle clearance technique, changes of speed, and stride patterns during racing (Iskra and Coh, 2011). However, there are no studies that have focused on the stride adjustment technique.

There are studies which quantified the stride adjustment technique during the takeoff in jumping events, such as the long jump and pole vault. Lee et al. (1982) showed that standard deviation of the ground contact position of the plurality of trials during the approach of the long jump athlete gradually increased from the start, reached a maximum value at a point in the latter half of the approach, and then decreased until the takeoff. Subsequently, the position where the maximum value of this standard deviation appears was defined as the position where the athlete starts stride adjustment. Hay (1988) also revealed that the maximum value of standard deviation of the ground contact position of the high level long jump athlete appeared earlier than that of the low level athlete. Similarly, it should be possible to clarify the stride adjustment pattern until the takeoff during the approach of the 400 $\mathrm{m}-\mathrm{H}$ event using the standard deviation of the ground contact position between multiple trials.

Based on the aforementioned method, this research aimed to clarify stride adjustment in the approach of the $400 \mathrm{~m}-\mathrm{H}$, and to examine the relationship with $400 \mathrm{~m}-\mathrm{H}$ performance.

\section{Methods}

\section{Participants}

Seven male 400-m hurdlers (body height 1.74 $\pm 3.39 \mathrm{~m}$, body mass $63.5 \pm 2.24 \mathrm{~kg}$ ) volunteered for this study. The performance index for each participant was their $400 \mathrm{~m}-\mathrm{H}$ personal best (400 mHPB; $53.66 \pm 1.21 \mathrm{~s})$. In addition, the $400-\mathrm{m}$ running personal best $(400 \mathrm{mPB} ; 50.58 \pm 1.55 \mathrm{~s}$ ) was used as an indicator of the running ability in the race without hurdles. The ratio of $400 \mathrm{mHPB}$ and $400 \mathrm{mPB}$ was used as an indicator of the running technique of the $400 \mathrm{~m}-\mathrm{H}(400 \mathrm{~m} / 400 \mathrm{mH}$; $0.94 \pm 0.02$ ), independent of the running ability of $400-\mathrm{m}$ distance. The index of the $400 \mathrm{~m}-\mathrm{H}$ technique in this study was slightly inferior to the value calculated from the world and European top athlete records $(0.96 \pm 0.01: \mathrm{n}=19)$ (Iskra and Pietrzak, 2016). The participants were informed of the purpose and contents of this experiment, and the study was carried out after obtaining their consent.

\section{Measures}

The measurements were obtained during the competitive season. Participants warmed up and then ran three times from the start to the second hurdle. In the trial, we encouraged participants to imagine the actual race. Participants were instructed not to change the position of the starting block among each trial, and sufficient rest was ensured between trials. The participants were recorded from one arbitrary arc center of the curved track by one high speed camera (EXILIM EX - 100F, manufactured by CASIO Corporation) so that the running direction of the participant was perpendicular to the optical axis of the camera (shooting speed: 240 frames/s). In addition, to improve panning accuracy, the running lane was the most inside one, which was closest to the arc center of the track (the radius of curvature was $37.9 \mathrm{~m}$ ). Prior to panning and recording, markers were set at $2.5-\mathrm{m}$ intervals inside the lane. In order to construct a twodimensional coordinate system on a curved track, markers were set at $2.5-\mathrm{m}$ intervals on both sides of the lane at the first hurdle installation point (45$\mathrm{m}$ from the start). A $2.05-\mathrm{m}$ pole was used to calibrate the aspect ratio for vertical and horizontal measurements. This calibration was performed at the center of the lane, $45 \mathrm{~m}$ from the starting point.

\section{Procedures}

The panned video was imported into a personal computer. The toe at the moment of landing at each step and the left greater trochanter at the moment of landing at each step were digitized using a video motion analysis system (Frame-DIAS V, DKH).

Moreover, it was assumed that the distance between the camera and the participant during panning was constant, and that the optical axis of the camera was orthogonal to the participants' running direction. Based on the calibration performed near the first hurdle, the coordinates of the measurement points in each frame during panning were calculated using the twodimensional four-point real length conversion method. Furthermore, based on the position of the 
marker inside the corner, the coordinates of the measurement points in each frame were converted to coordinates with the start point as a reference (Ohnuma et al., 2014). To confirm the accuracy of the two-dimensional coordinate construction, the coordinates of the markers of each frame of the video were estimated, and errors with the coordinates of the actual markers were investigated. As a result, the error in the horizontal direction with respect to the markers installed at 2.5-m intervals near the first hurdle was $0.019 \mathrm{~m}$ on average. It was lower than the error of a previous study (Ohnuma et al., 2014), and it was considered that the reconstruction of two dimensional coordinates in this study was sufficiently accurate.

\section{Variables}

The variables in this study were as follows.

- Standard deviation of toe-hurdle distance (SDTH): Standard deviation of the distance from the toe to the first hurdle in each step among the three trials. It was considered an indicator of the accumulated error of stride in the approach.

- SDTHmax: Maximum value of the SDTH.

- SDTHmax appearance position: Distance to the hurdle in the step where SDTHmax appeared. This was defined as the point where the athlete started stride adjustment.

Adjustment of Stride Length: The rate of change of SDTH at each step after the appearance of SDTHmax. This was calculated from the following formula and was considered the stride adjustment rate at each step. "' was the step number of the approach. In addition, the SDTH at step $0(\mathrm{i}=0)$ was expressed as SDTHT0.

Adjustment of Stride Length $(\mathrm{i})=\{-(\mathrm{SDTHi}-$ SDTHi-1)/(SDTHmax - SDTHT0) $\} \times 100$

- Cumulative Adjustment of Stride Length: The sum of Adjustment of Stride Length at each step after appearance of SDTHmax.

- Standard deviation of stride length (SDSL): The stride was obtained from the difference of the toe ground contact position of each step, and it was considered the standard deviation between trials of each stride.

- Rate of velocity deceleration: The product of the lateral displacement of the left trochanter and the time required for the grounding of one toe to the other toe, which was considered the running velocity in each step (this value was the average of three trials). From the maximum value of the running velocity of each step, the decreasing rate of running velocity in step 0 was considered the rate of velocity deceleration in the approach section.

\section{Statistical analysis}

Pearson's correlation coefficients were calculated to examine the relationship between each variable, $400 \mathrm{mHPB}$, and $400 \mathrm{~m} / 400 \mathrm{mH}$. The significance level was set at $\mathrm{p}<0.05$.

\section{Results}

Figures 1 and 2 show changes in SDTH and SDSL throughout the approach. The number of steps from the start to the takeoff did not change between trials in any participants $(22.29 \pm 0.45$ steps). All participants reached the maximum speed in the approach, and the speed decreased until just before the takeoff. The average value of the SDTH of the participants gradually increased after the start, reached the maximum value in the latter half of the approach section $(17.21 \pm 6.99 \mathrm{~m}$ as the distance from the hurdle), and then decreased until the takeoff (Figure 1). SDSL also fluctuated up to $0.18 \mathrm{~m}$ throughout the approach. This SDSL increased from immediately after the start, and it tended to increase remarkably from 4 steps before the takeoff (Figure 2). Figure 3 shows change in the cumulative stride adjustment rate from the previous 4 steps before the takeoff. The step where the increase in the cumulative stride adjustment rate was the largest was two steps before the takeoff, and the standard deviation was also the largest here.

Table 1 shows the relationships between the index of the stride adjustment technique, $400 \mathrm{~m}$ $\mathrm{HPB}, 400 \mathrm{~m} / 400 \mathrm{mH}$, and the rate of velocity deceleration. There was a significant negative correlation between SDTHmax, Mean-SDSL and the $400 \mathrm{~m} / 400 \mathrm{mH}$ (Figures 4 and 5). There was a significant positive correlation between the rate of velocity deceleration and SDTHmax (Table 1). There were no significant correlations between other variables. 


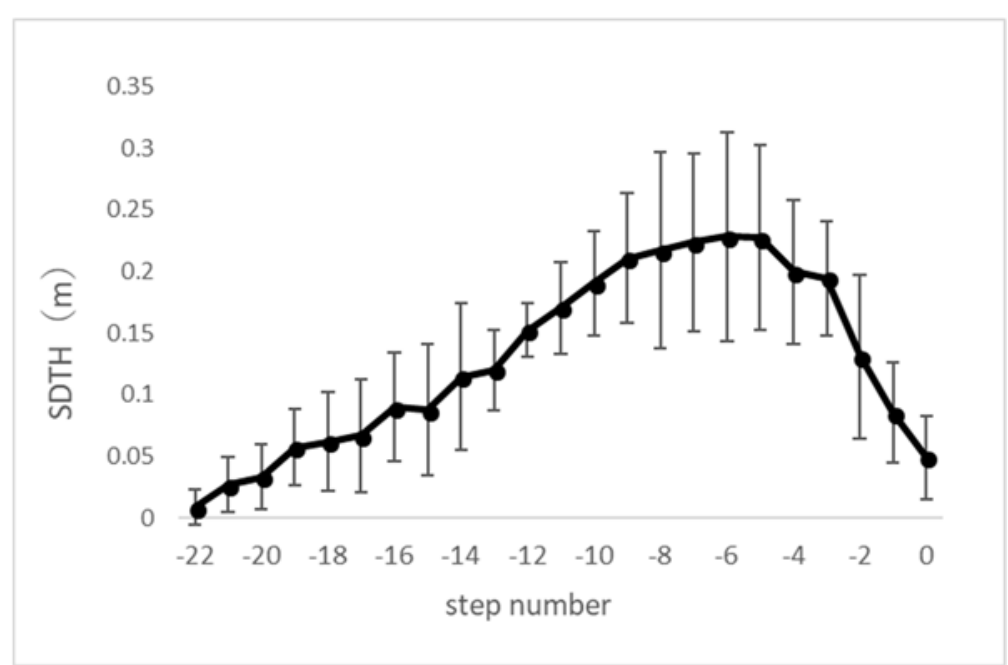

Figure 1

Change in SDTH

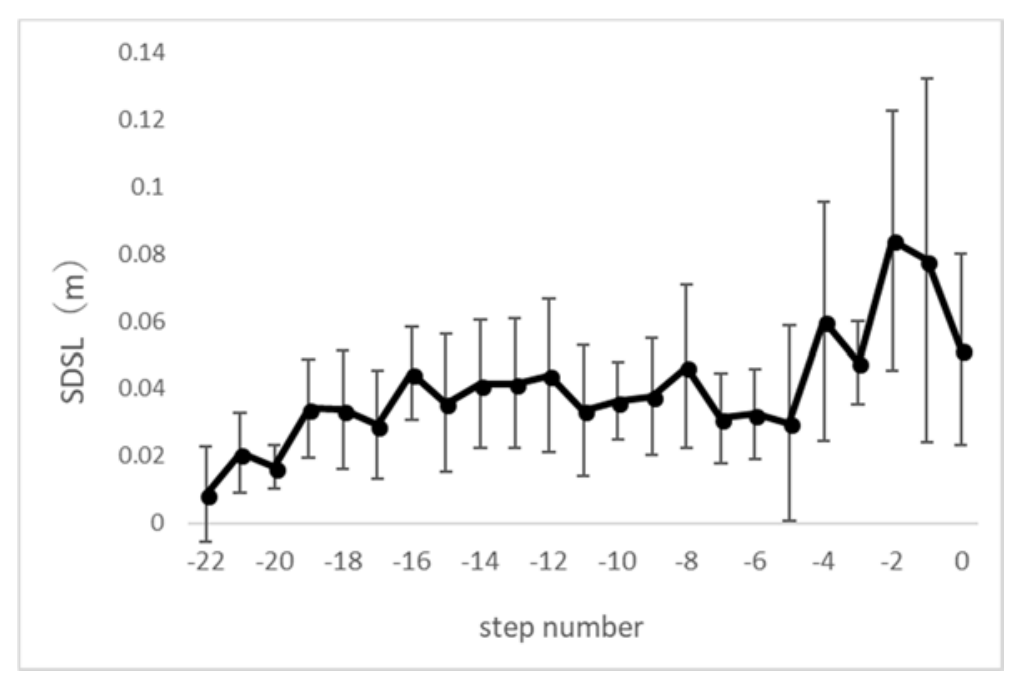

Figure 2

Change in SDSL 


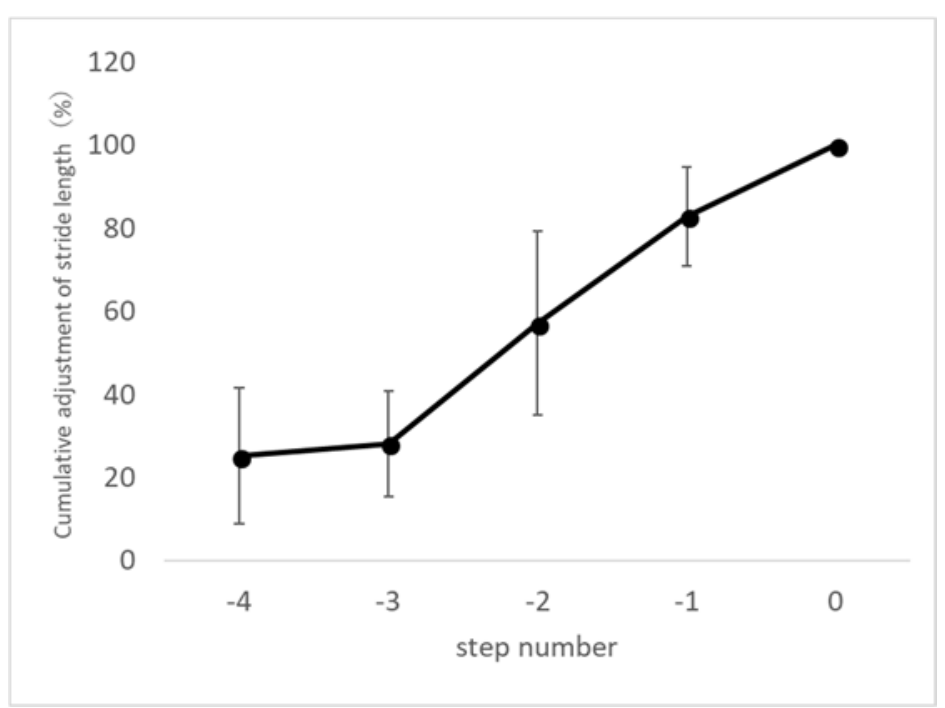

Figure 3

Changes in cumulative adjustment of stride length 4 steps before the takeoff

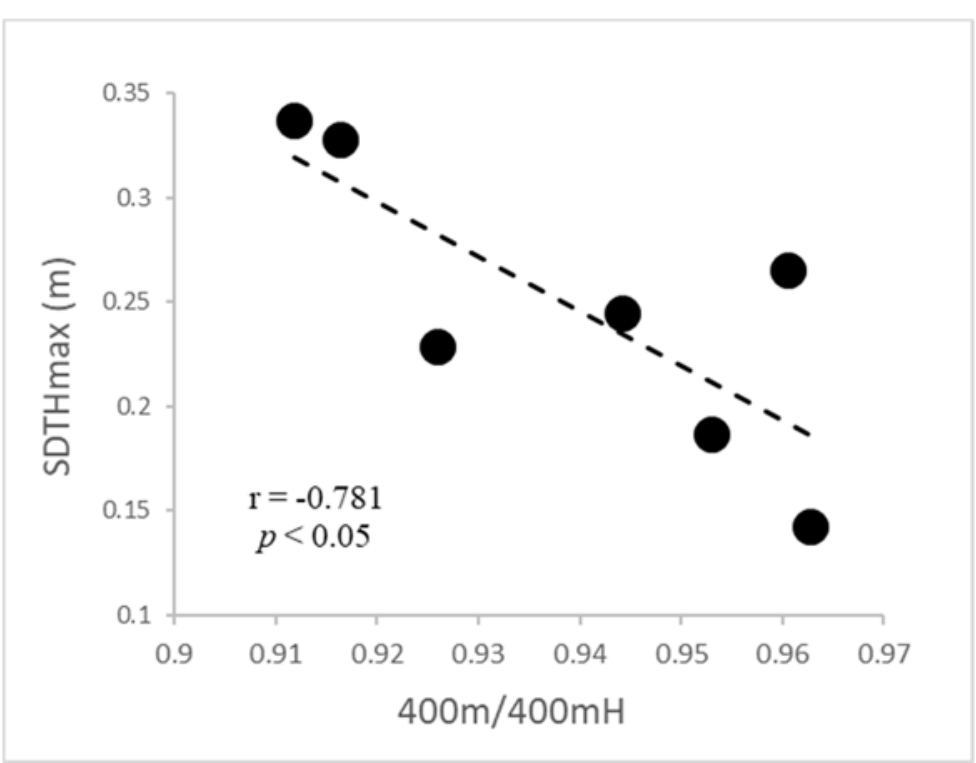

Figure 4

Relationship between SDTHmax and $400 \mathrm{~m} / 400 \mathrm{mH}$ 


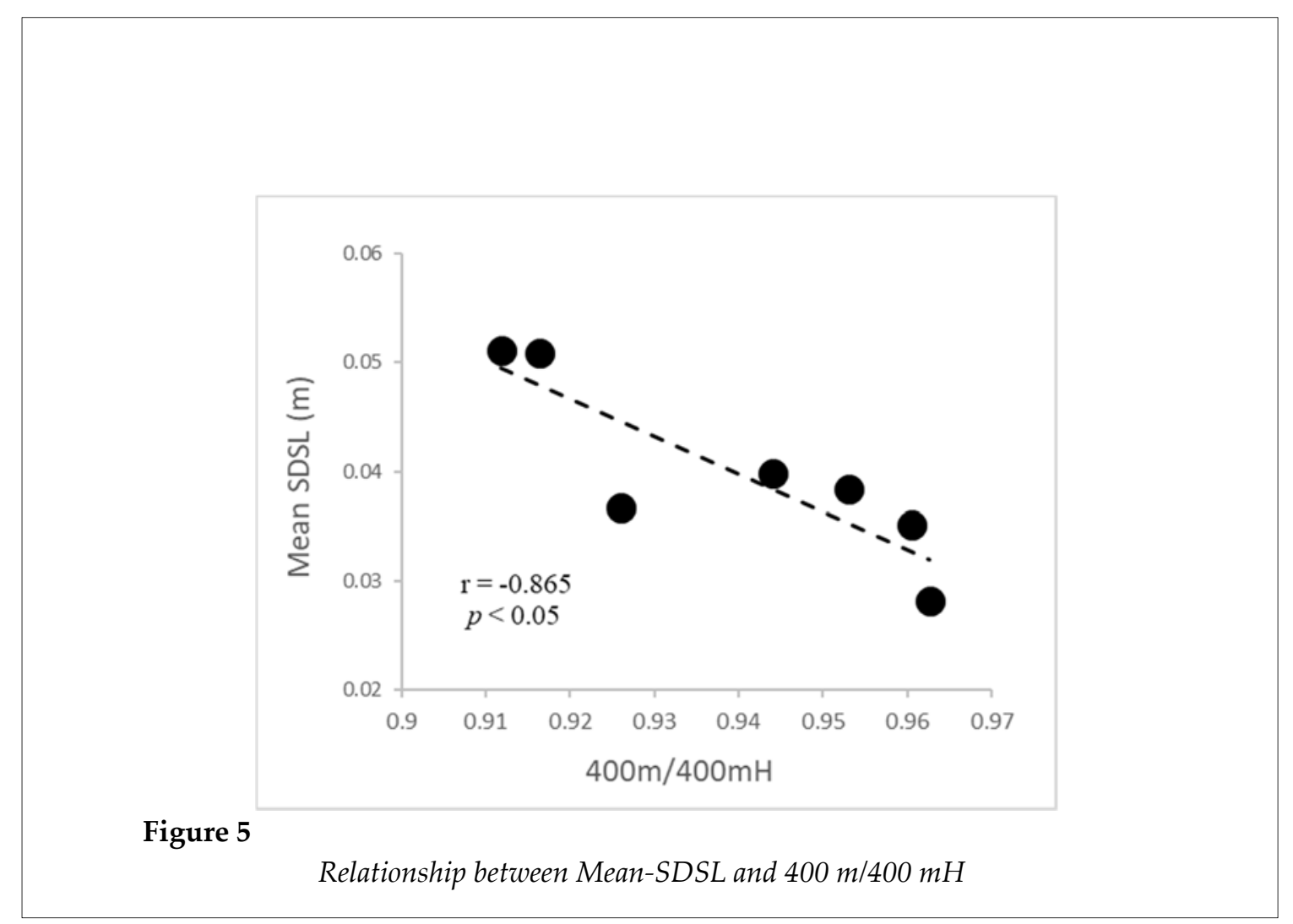

Table 1

Relationship between the index of the stride adjustment technique, $400 \mathrm{mHPB}, 400$ $\mathrm{m} / 400 \mathrm{mH}$, and the rate of velocity deceleration

\begin{tabular}{|c|c|c|c|c|c|c|}
\hline & Mean \pm SD & Max & Min & $400 \mathrm{mHPB}$ & $\begin{array}{l}400 \mathrm{~m} / 400 \\
\mathrm{mH}\end{array}$ & $\begin{array}{c}\text { Rate of } \\
\text { velocity } \\
\text { deceleration } \\
(\%) \\
\end{array}$ \\
\hline 400mHPB & $53.66 \pm 1.21$ & $\begin{array}{c}55.3 \\
8\end{array}$ & $\begin{array}{c}51.6 \\
7\end{array}$ & 1.00 & -.068 & .491 \\
\hline $400 \mathrm{mPB}$ & $50.58 \pm 1.55$ & $\begin{array}{c}53.1 \\
9\end{array}$ & $\begin{array}{c}48.9 \\
2\end{array}$ & $.714^{*}$ & .650 & .056 \\
\hline $400 \mathrm{~m} / 400 \mathrm{mH}$ & $0.94 \pm 0.02$ & 0.96 & 0.91 & -.068 & 1.00 & -.451 \\
\hline Mean-SDTH (m) & $0.13 \pm 0.02$ & 0.17 & 0.09 & .486 & -.682 & .653 \\
\hline SDTHmax (m) & $0.25 \pm 0.07$ & 0.34 & 0.14 & .410 & $-.781^{*}$ & $.791^{*}$ \\
\hline SDTHmax appearance position (m) & $17.21 \pm 6.99$ & $\begin{array}{c}32.4 \\
6\end{array}$ & $\begin{array}{c}11.6 \\
1\end{array}$ & -.464 & -.190 & -.154 \\
\hline Mean-SDSL (m) & $0.04 \pm 0.01$ & 0.05 & 0.03 & .095 & $-.865^{*}$ & .607 \\
\hline $\begin{array}{l}\text { Cumulative adjustment of stride length } \\
\text { at step }-4(\%)\end{array}$ & $25.13 \pm 16.34$ & $\begin{array}{c}48.0 \\
4\end{array}$ & 5.73 & -.326 & -.664 & -.016 \\
\hline $\begin{array}{l}\text { Cumulative adjustment of stride length } \\
\text { at step }-3(\%)\end{array}$ & $28.20 \pm 12.67$ & $\begin{array}{c}41.1 \\
9\end{array}$ & $\begin{array}{c}10.3 \\
7\end{array}$ & .025 & -.540 & .387 \\
\hline $\begin{array}{l}\text { Cumulative adjustment of stride length } \\
\text { at step }-2(\%)\end{array}$ & $57.11 \pm 22.13$ & $\begin{array}{c}92.8 \\
4\end{array}$ & $\begin{array}{c}37.5 \\
4\end{array}$ & -.023 & -.578 & -.118 \\
\hline $\begin{array}{l}\text { Cumulative adjustment of stride length } \\
\text { at step }-1(\%)\end{array}$ & $82.83 \pm 11.86$ & $\begin{array}{c}95.3 \\
1\end{array}$ & $\begin{array}{c}57.7 \\
1\end{array}$ & .610 & .142 & -.160 \\
\hline Rate of velocity deceleration (\%) & $4.93 \pm 2.22$ & 8.16 & 0.86 & .491 & -.451 & 1.00 \\
\hline
\end{tabular}




\section{Discussion}

\section{Stride adjustment in the approach section}

SDTH, an indicator of the accumulated error of the stride and state of modification in the approach section, gradually increased after the start and tended to decrease on average from the sixth step (mean $15.09 \mathrm{~m}$ ) to the takeoff. This was the same trend as shown in previous studies in long jump, triple jump, and pole vault athletes (Bradshaw et al., 2006; Hay and Koh, 1988; Scott et al., 1997; Tamura et al., 2012). Additionally, SDSL represents stride variation for each step, which increased after the start and tended to increase greatly from the fourth step. The cumulative adjustment of the stride length, that represents which steps and how much adjustment of stride length was completed after SDTHmax appearance, was markedly increased in the second and first steps.

It was important to minimize the accumulation of errors in the stride to acquire a high takeoff velocity in the approach at the long jump; however, it was difficult to implement this perfectly (Hay, 1988). Furthermore, it was considered that while SDSL in the first half of the approach was accidental, with the variation resulting from running for the purpose of accelerating, SDSL that appeared from the midfield to the takeoff had a strategic variation to correct the accumulation of stride errors in the first half of the approach (Tamura et al., 2012).

In this study, even for participants with the smallest SDTHmax of $0.14 \mathrm{~m}$, deceleration was observed from reaching the maximum speed just before the takeoff. There was also a significant positive correlation between SDTHmax and the rate of velocity deceleration. From these trends of SDTH and SDSL, it was inferred that it was difficult for the athletes to run without increasing the stride error accumulation, even in the approach of the $400 \mathrm{~m}-\mathrm{H}$. In addition, it was suggested that athletes seemed to adjust their stride from the middle stage of the approach to the latter half by sensing stride error accumulation in the middle of the approach. The strides just before the takeoff were greatly involved in stride adjustment. In the process, velocity deceleration occurred, and it was shown that the higher the accumulation error of stride, the greater the deceleration in the approach section.
The relationship between stride adjustment technique and performance

In elite long jump athletes, the point at which there is the maximum accumulation of the stride error appears early (Hay, 1988). Moreover, low-level pole vault athletes have a lower cumulative adjustment of stride length from the appearance of the maximum value of stride error accumulation to two steps before the takeoff compared to high level athletes. Furthermore, most of the stride adjustment occurs two steps before the takeoff (Tamura, 2012). These results show importance of the ability to sense the accumulation of stride error early and perform stride adjustments in the approach during jumping events.

In this study, there was no significant correlation between the SDTHmax appearance position and $400 \mathrm{mHPB}$ or $400 \mathrm{~m} / 400 \mathrm{mH}$. Furthermore, since the degree of the completion of stride adjustment in the second step was the most uneven among individuals, it was predicted that there were differences depending on the performance level. However, no significant correlation was found in any participant. In contrast, SDTHmax and Mean-SDSL showed a significant correlation with the $400 \mathrm{~m} / 400 \mathrm{mH}$. From these findings, it may be assumed that a good technique can reduce the variation of the stride by step and the maximum accumulation of stride error, and that performing the $400 \mathrm{~m}-\mathrm{H}$ is not influenced by the sprint capacity of a 400-m event. This SDTHmax was significantly correlated with the rate of velocity deceleration. Therefore, the ability to reduce maximum accumulation stride error was considered to contribute to maintaining the velocity up to the takeoff. This seems to explain the relationship between SDTHmax and $400 \mathrm{~m} / 400 \mathrm{mH}$. However, there was no significant correlation between the rate of velocity deceleration and $400 \mathrm{~m} / 400 \mathrm{mH}$. The reason speculated is that the rate of velocity deceleration was only for the approach section and that the hurdle clearance technique was influenced at $400 \mathrm{~m} / 400 \mathrm{mH}$. On the other hand, Mean-SDSL and SDTHmax evaluated in the approach section may affect the stride change and the rate of velocity deceleration for up to all ten hurdles in the race. Therefore, the association between SDTHmax, Mean-SDSL, and $400 \mathrm{~m} / 400$ 
$\mathrm{mH}$ was thought to be stronger. research

Practical implications and directions of future

In this study, the higher the SDTHmax, the greater the rate of velocity deceleration to the hurdle and the lower $400 \mathrm{~m} / 400 \mathrm{mH}$. Therefore, in training it is necessary to focus on reducing accumulation stride error. It can be said that the stride fluctuation and the velocity deceleration after the SDTHmax appearance position are the result of the run of the previous part. Therefore, after SDTHmax increases, it is inferred that it is too late for the hurdler to run without causing stride fluctuations and velocity deceleration. In other words, the importance of training with a focus on the first half of the run to the hurdle was suggested. The higher the running efficiency of the $400 \mathrm{mH}$, the lower Mean SDSL and SDTHmax will be.

The index of the stride adjustment technique evaluated in this research was based only on the approach. However, from these results, the stride adjustment technique evaluated in the approach section was considered to relate to the smooth running of the entire $400 \mathrm{~m}-\mathrm{H}$ race. The $400 \mathrm{~m}$-HPB of participants varied from 55.38 to 51.67 in this study. Therefore, there is a limit to applying all the results of this study to worldclass hurdlers and beginners. Furthermore, no evaluation of hurdle clearance techniques has been conducted in this study. In the future, it would be necessary to analyze hurdlers of an even wider performance level and consider hurdle clearance technique.

\section{Acknowledgements}

This study was conducted in the Hiroshima University, Japan. The authors received no external funding, and have no conflict of interest to disclose.

\section{References}

Bradshaw EJ, Aisbett B. Visual Guidance during Competition Performance and Run-through Training in Long Jumping. Sport Biomech, 2006; 5: 1-14

Ditroilo M, Marini M. Analysis of the race distribution for male $400 \mathrm{~m}$ hurdlers competing at the 2000 Sydney Olympic Games. New Studies in Athletics, 2001; 16: 15-30

Hay JG. Approach strategies in the long jump. Int J Sport Biomech, 1988; 4: 114-129

Hay JG, Koh TJ. Evaluating the Approach in the Horizontal Jumps. Int. J Sport Biomech, 1988; 4: 372-392

Iskra J, Čoh M. Biomechanical studies on running the 400 m hurdles. Hum Mov, 2011; 12: 315-323

Iskra J, Pietrzak M. Flat versus rhythm endurance in $400 \mathrm{~m}$ hurdle training. Atletika, 2016; 39-44

Karube S, Hirai T, Watanabe C. Research in the subjective estimation that Athlete in 400mHurdles. Bulletin of sports and physical education research center, Hosei University, 2003; 21: 15-21

Lee DN, Lishman JR, Thomson JA. Regulation of gait in long jumping. Journal of Experimental Psychology: Human perception and performance, 1982; 8: 448-459

Morioka Y, Enomoto Y, Sugita M, Matsua A, Ae M, Kobayashi K. Analysis of the race-pattern for elite male 400-m hurdlers. Jpn J Biomechanics Sports Exercise, 2005; 9: 196-204

Nagasawa M. An Analysis of the 400m Hurdle Race for Novices. Memoirs of the Faculty of Education, Akita University. Educational science, 1995; 48: 49-60

Ohnuma H, Hirano Y, Tachi M. The changes of left and right leg movement on curved path during the latter half of 400m sprint. Jpn J Phys Fitness Sports Med, 2014; 63: 269-278 
Scott MA, Li F, Davids K. Expertise and the regulation of gait in the approach phase of the long jump. J Sports Sci, 1997; 15: 597-605

Tamura Y, Yuasa K, Ishimura K, Usui S. Regulation of stride length during the approach run in the pole vault. Japan J Phys Educ Hlth Sport Sci, 2012; 57: 47-57

Yasui T, Hondo S, Rui T. Aoyama K, Ichikawa D, Endo T. The study of race analysis about each performance level in $400 \mathrm{~m}$ hurdle sprint. Research quarterly for athletics, 2008; 75: 12-20

\section{Corresponding author:}

\section{Yusuke Ozaki}

Address: 1-1-1, Kagamiyama, Higashi-Hiroshima City, Hiroshima, Japan, 739-8524

Phone number: +81-90-1973-3240

Fax number: +81-8-2424-3478

E-mail: d185353@hiroshima-u.ac.jp 